\title{
VINHOS TINTOS DO NOROESTE PAULISTA: RELAÇÃO ENTRE OS PERFIS FÍSICO-QUÍMICO E SENSORIAL
}

Artigo submetido em outubro/2011 e aceito em agosto/2012

\section{RESUMO}

Os vinhos tintos, no Brasil, são muito apreciados pelos consumidores das regiões de clima tropical, pelo fato de apresentar tipicidade aromática e paladar frutado. Nesse sentido, o objetivo desse artigo é verificar a qualidade de três vinhos tintos provenientes de vinícolas da região noroeste do estado de São Paulo, através da relação entre determinações físico-químicas e atributos sensoriais. Três vinhos dessa região foram avaliados por meio de métodos analíticos e sensoriais e foi possível observar diferenças significativas nas determinações acidez fixa, teor alcoólico, teor de fenólicos totais e extrato seco. A análise multidimensional mostrou a interação do teor de fenólicos totais com a adstringência, a relação do extrato seco total com o corpo da bebida a relação da acidez volátil na avaliação do odor das amostras. Foi possível constatar a influência físico-química na avaliação dos atributos sensoriais, evidenciando a importância do perfil químico na elucidação de possíveis alterações sensoriais nos vinhos tintos.

PALAVRAS-CHAVE: vinhos tintos, vinificação, avaliação sensorial, propriedades físico-químicas.

\section{RED WINES FROM NORTHWEST OF SÃO PAULO STATE: RELATIONSHIP BETWEEN PHYSICOCHEMICAL AND SENSORY APPROACHES}

\section{ABSTRACT}

Brazilian red wines are very appreciated by consumers from tropical regions, because they typically have aromatic and fruity taste. Thus, the aim of this article is to verify the quality of red wines from wineries located in Northwest region of São Paulo, based on the relationship between physicochemical properties and sensory attributes. Three red wines from this region was evaluated by sensory and analytical methods and it was possible to observe significant differences in the fixed acidity, alcoholic content, total phenolic content and total dry extract. Multidimensional Scaling showed relationship between total phenolic content and astringency, total dry extract and body and the relationship of volatile acidity in the assessment of odor samples. It was possible to see the physicochemical influence in sensory evaluation, indicating the importance of the chemical profile in the elucidation of possible sensory alterations in red wines.

KEY-WORDS: red wines, winemaking, sensory evaluation, physicochemical properties. 


\section{VINHOS TINTOS DO NOROESTE PAULISTA: RELAÇÃO ENTRE OS PERFIS FÍSICO-QUÍMICO E SENSORIAL}

\section{INTRODUÇÃO}

Os vinhos de mesa representam o maior volume de vinhos comercializados no Brasil e são produzidos nos estados do Rio Grande do Sul, Santa Catarina, Paraná, São Paulo e Minas Gerais, destacando-se o estado do Rio Grande do Sul com cerca de $90 \%$ da produção (MELLO, 2002). Contrastando com esses dados, verifica-se a difusão de produção de uvas, vinhos e seus derivados em regiões emergentes do Brasil, desde a metade sul do Rio Grande do Sul, Noroeste paulista e região Nordeste. Essa ascensão se deve à diversidade de clima e solos dessas regiões que possibilitam a produção de vinhos com características peculiares e diferenciadas, adaptadas aos diferentes paladares dos consumidores brasileiros (GUERRA et al., 2009).

Nesse contexto, essas regiões de clima tropical estão se destacando no cultivo de uvas rústicas da espécie Vitis labrusca, visto que tais cultivares possibilitam a programação da colheita para qualquer época do ano, eliminando períodos de entressafra e promovendo alta rentabilidade. Esses fatores foram fundamentais para caracterizar o pioneirismo da região do vale do São Francisco na produção de uvas como alternativa para a elaboração de vinhos de mesa regionais (CAMARGO, 2004). Os vinhos produzidos nas regiões tropicais não são conhecidos mundialmente e sua produção é pequena quando comparada à produção de vinhos mundial. Assim, necessita-se aprimoramento tecnológico para se garantir um vinho de qualidade com o objetivo de conquistar espaço e competitividade no mercado (TONIETTO; CAMARGO, 2006).

A qualidade de um vinho está sob influência de fatores como as condições sanitárias da uva, a tecnologia de vinificação utilizada (LEE et al., 2006), o tipo do solo, as condições climáticas e o manejo da videira (CHAVARRIA et al., 2008). Esses fatores são responsáveis por determinar as propriedades químicas do vinho e proporcionar características sensoriais de qualidade. As principais substâncias químicas que compõem o vinho são açúcares, álcoois, ácidos orgânicos, sais de ácidos minerais e orgânicos, compostos fenólicos, compostos nitrogenados, substâncias voláteis e aromáticas e substâncias que promovem a turbidez da bebida como pectinas e gomas (JACKSON, 2008; HASHIZUME, 1983).

Estudos científicos mostram que a qualidade de um vinho tinto é baseada na avaliação de atributos sensoriais e que as propriedades físico-químicas são determinadas somente para explicar possíveis alterações sensoriais detectadas nos vinhos (CHIRA et al., 2011). No entanto, a relação entre os atributos sensoriais e o perfil físico-químico dos vinhos é um dos objetivos principais das pesquisas científicas da área enológica (GIRARD et al., 2001), buscando entender quais determinações físico-químicas influenciam os atributos sensoriais e de que forma essa influência é caracterizada (THORNGATE, 1997).

As metodologias mais aplicadas, atualmente, para o estudo da relação entre as propriedades físico-químicas e os atributos sensoriais são os métodos estatísticos multivariados como a Análise de Componentes Principais, Análise Multidimensional e Clustering Analysis. Além disso, metodologias mais complexas como a Análise Descritiva Quantitativa (ADQ) também são empregadas na avaliação dos atributos sensoriais de bebidas complexas como o vinho (CAMPO et al., 2010; ORTEGA-HERAS et al., 2010). 
Com o objetivo de enriquecer os estudos das relações entre o perfil físico-químico e sensorial dos vinhos tintos, o objetivo desse artigo foi relacionar as características físico-químicas de três vinhos com seus perfis sensoriais aplicando a análise multidimensional e a análise de cluster.

\section{MATERIAL E MÉTODOS}

\section{Determinações analíticas}

Os vinhos tintos de mesa utilizados na pesquisa foram adquiridos em comércios locais da cidade de São José do Rio Preto, atendo-se ao fato de que cada um deles pertencia a uma vinícola diferente da região noroeste do estado de São Paulo. Foram analisados dois vinhos tintos secos de mesa: A com 10,7\% de álcool (v/v) e B com 10,8\% de álcool (v/v); e um vinho tinto fino seco $\mathrm{F}$ com $11,5 \%$ de álcool $(\mathrm{v} / \mathrm{v})$, sendo esses teores alcoólicos especificados em rótulo. As metodologias empregadas e os materiais utilizados para a determinação das propriedades físicoquímicas estão expostos na Tabela 1.

Tabela 1: Metodologias empregadas para as determinações físico-químicas.

\begin{tabular}{lcc}
\hline \multicolumn{1}{c}{$\begin{array}{c}\text { Determinação } \\
\text { analítica }\end{array}$} & Materiais & Metodologia \\
\hline Acidez total (ACT) & Titulometria & BRASIL (1986); IAL (2005) \\
Acidez volátil (ACV) & Titulometria & AOAC (1992); IAL (2005) \\
Extrato Seco (EXT) & Gravimetria & FOLIN; CIOCALTEAU (1927) \\
Fenólicos totais (FEN) & Espectrofotometria & BRASIL (1986); IAL (2005) \\
Sulfatos (SUL) & Método químico aproximativo de & AOAC (1992) \\
Açúcar redutor (ACR) & Marty & AOAC (1992); IAL (2005) \\
Álcool (ALC) & Titulometria & \\
\hline
\end{tabular}

A acidez fixa (ACF) foi determinada pela subtração da acidez total com a acidez volátil (IAL, 2005). Os dados referentes a todas as determinações físico-químicas foram coletados em triplicata, sendo analisados estatisticamente pelo teste não-paramétrico de Kruskal-Wallis com aplicação do teste de comparação múltipla de Dunn a 5\% de significância.

\section{Análise sensorial}

A análise sensorial dos vinhos foi caracterizada, primeiramente, pela aplicação de testes triangulares com o objetivo de realizar a triagem dos provadores que seriam selecionados para a avaliação comparativa das amostras (DUTCOSKY, 2011). Os provadores selecionados foram encaminhados para cabines individuais e submetidos a um questionário de caracterização inicial contendo a frequência de consumo da bebida. Após a aplicação desse questionário preliminar, os provadores avaliaram as amostras, sendo essas apresentadas de forma monádica, utilizando uma escala numérica com 11 pontos, variando de 0 a 10, sendo 0 o pior conceito e 10 o melhor conceito. Os atributos avaliados foram: acidez (ACD), corpo (COP), doçura (DOC), adstringência $(A D S)$, teor alcoólico $(A L C)$, cor $(C O R)$ e odor (ODR). Além disso, os provadores foram 
questionados sobre a frequência de consumo da bebida. As análises sensoriais foram realizadas no período da tarde, entre 14 e 17 horas, no Laboratório de Análise Sensorial do Instituto de Biociências, Letras e Ciências Exatas.

\section{Análise dos dados}

Os dados provenientes dos testes sensoriais foram submetidos à Análise de Variância com aplicação do teste de comparação múltipla de Tukey e os dados referentes às determinações físico-químicas foram avaliados pelo teste de Kruskal-Wallis, seguido do teste de comparação múltipla de Dunn. Além disso, a relação entre as determinações físico-químicas e os atributos sensoriais foi avaliada pela aplicação da Análise de Cluster seguida da Análise Multidimensional (CASTILHOS et al., 2012). Todos os testes foram realizados ao nível de significância de 5\%, utilizando o software Statistica 7

\section{RESULTADOS E DISCUSSÃO}

\section{Determinações analíticas}

Os resultados das determinações analíticas dos vinhos avaliados no estudo encontram-se expostos na Tabela 2.

Tabela 2: Medianas (amplitudes) das determinações analíticas.

\begin{tabular}{lcccc}
\hline Determinações $^{* *}$ & Vinho A & Vinho B & Vinho F & Valor P \\
\hline ACT (meq/L) & $91,28(2,95)$ & $91,27(2,95)$ & $81,46(3,92)$ & 0,063 \\
ACV (meq/L) & $10,79(1,96)$ & $14,72(1,97)$ & $14,72(2,95)$ & 0,095 \\
ACF (meq/L) & $80,49^{\mathrm{a}}(0,99)$ & $78,52^{\mathrm{ab}}(2,95)$ & $70,67^{\mathrm{b}}(6,87)$ & $\mathbf{0 , 0 2 6}$ \\
EXT (g/L) & $18,00^{\mathrm{ab}}(0,40)$ & $12,00^{\mathrm{b}}(7,20)$ & $43,20^{\mathrm{a}}(19,60)$ & $\mathbf{0 , 0 4 6}$ \\
FEN (g/L) & $0,965^{\mathrm{b}}(0,098)$ & $1,120^{\mathrm{ab}}(0,083)$ & $1,230^{\mathrm{a}}(0,058)$ & $\mathbf{0 , 0 2 7}$ \\
ACR (g/L) & $2,07(0,13)$ & $1,66(0,14)$ & $1,69(0,14)$ & 0,066 \\
ALC (\% v/v) & $11,60^{\mathrm{a}}(0,90)$ & $9,90^{\mathrm{b}}(0,60)$ & $10,40^{\mathrm{ab}}(0,20)$ & $\mathbf{0 , 0 2 6}$ \\
SUL (g/L) & entre 0,7 e 1 & menor que 0,7 & menor que 0,7 & - \\
\hline
\end{tabular}

"Método semiquantitativo - teste aproximativo de Marty.

${ }^{* *}$ Medianas seguidas por letras distintas na mesma linha diferem entre si pelo teste de Dunn a $5 \%$ de significância. ACT: acidez total, ACV: acidez volátil, ACF: acidez fixa, EXT: extrato seco, SUL: sulfatos, FEN: fenólicos totais, ACR: açúcares redutores, ALC: teor alcoólico.

De acordo com os resultados da Tabela 2 foi possível constatar a existência de diferenças significativas nas seguintes determinações: acidez fixa $(P=0,026)$; extrato seco $(P=0,046)$; fenólicos totais $(P=0,027)$ e teor alcoólico $(P=0,026)$. O vinho fino seco (vinho $F)$, de acordo com o teste de Dunn, apresentou menores valores de acidez fixa em relação ao vinho de mesa $A$, maiores valores de extrato seco em relação ao vinho de mesa $B$ e maiores valores de fenólicos totais quando comparados ao vinho de mesa $A$.

A acidez volátil é caracterizada pelos ácidos orgânicos voláteis como ácido acético, fórmico, butírico, propiônico, entre outros. Esses ácidos são formados durante o processo fermentativo e são responsáveis por determinar a sanidade da bebida. Por isso, espera-se que os índices de acidez volátil sejam baixos, caracterizando a qualidade do vinho. A acidez fixa, por sua vez, está vinculada aos ácidos tartárico, málico, lático, succínico e cítrico, muitos deles presentes 
na própria uva e outros provenientes do processo de fermentação e estabilização (JACKSON, 2008). Esses ácidos compõem o vinho e são responsáveis por sua estrutura, promovendo maior corpo à bebida (AMERINE et al., 1967).

Em todas as amostras, os valores de acidez fixa e volátil apresentaram-se de acordo com o preconizado pela legislação, sendo mínimo de $55 \mathrm{meq} / \mathrm{L}$ e máximo de $130 \mathrm{meq} / \mathrm{L}$ para acidez total e valores de até $20 \mathrm{meq} / \mathrm{L}$ para acidez volátil (BRASIL, 2004). Por conseguinte, os valores de acidez fixa também apresentaram-se em conformidade com o esperado. $O$ menor teor de acidez fixa verificado no vinho fino pode estar vinculado ao processo de vinificação de cada vinícola e, principalmente, à composição da uva, visto que vinhos finos são produzidos por uvas da espécie Vitis vinifera e vinhos de mesa pelas espécies Vitis labrusca.

O extrato seco total refere-se ao peso do resíduo resultante da evaporação de compostos voláteis. Esse resíduo é composto de carboidratos, glicerinas, ácidos não-voláteis, compostos nitrogenados, taninos, álcoois superiores e minerais. Está vinculado à maturação da uva, ao processo de vinificação e aos componentes responsáveis pelo corpo do vinho (JACKSON, 2008). Segundo Zoecklein et al. (1994), o vinho seco com teor de extrato seco inferior a $20 \mathrm{~g} / \mathrm{L}$ apresenta-se leve ao paladar; em contrapartida, o vinho com $30 \mathrm{~g} / \mathrm{L}$ ou mais é considerado como encorpado. A discrepância observada nos resultados dessa determinação era esperada, visto que os vinhos tintos finos são considerados mais encorpados que os vinhos de mesa devido à solubilização das substâncias sólidas da uva na etapa de maceração que, por sua vez, na vinificação de uvas viníferas, é mais prolongada (RIZZON, 2006).

Os compostos fenólicos presentes no vinho fino foram superiores em relação aos vinhos de mesa analisados no estudo. Como os polifenois estão relacionados à estrutura da coloração do vinho e ao sabor adstringente, era previamente esperado que o vinho tinto fino apresentasse teor de fenólicos totais superior aos vinhos de mesa (AMERINE; OUGH, 1986). Os compostos fenólicos são influenciados pelo teor alcoólico do vinho, visto que o aumento de etanol durante a fermentação alcoólica resulta na desnaturação progressiva de pigmentos devido à ruptura de pontes de hidrogênio entre os compostos fenólicos, transformando-os em substâncias incolores (JACKSON, 2008). Nesse contexto, verificou-se, pelos resultados, que o vinho de mesa A que apresentou maior teor alcoólico também apresentou menor índice de fenólicos totais, reiterando a influência do etanol na desnaturação fenólica.

Todas as amostras apresentaram-se conformes à legislação em relação ao teor alcoólico, ou seja, apresentaram-se entre 8,6 e $14^{\circ} \mathrm{GL}$. Porém, todas elas não apresentavam a quantidade de álcool especificada no rótulo. As amostras de vinhos não apresentaram diferenças significativas em relação à acidez total, acidez volátil e açúcares redutores. Todas essas determinações estavam em conformidade com a legislação, assim como o teor de sulfatos das amostras que apresentou índices inferiores a 1,0 g/L.

\section{Análise sensorial}

Os testes triangulares foram aplicados em 30 provadores com o objetivo de verificar a capacidade de diferenciação de três amostras, sendo duas delas iguais. Desse total, somente 8 provadores conseguiram discriminar a amostra diferente, sendo esses os responsáveis pelos resultados da análise comparativa entre as amostras de vinho.

Com o objetivo de caracterizar a amostra de provadores, a frequência de consumo de vinho foi analisada e constatou-se que $50 \%$ deles às vezes consomem vinho; $25 \%$ consomem frequentemente; $12,5 \%$ sempre consomem e $12,5 \%$ raramente consomem vinhos. A preferência 
de consumo também foi questionada e os resultados mostraram que os vinhos $A(37,5 \%)$ e $B$ $(37,5 \%)$ foram os preferidos em relação ao vinho $F(25,0 \%)$. Além disso, o vinho $F$ apresentou a menor preferência dentre os provadores: $62,5 \%$ deles não optaram pelo vinho fino como preferido. Um fato interessante a ser abordado nesse estudo foi a intensa preferência dos provadores que frequentemente consomem vinhos pelo vinho fino. Em contrapartida, a maioria dos provadores que às vezes consomem vinhos preferiu os vinhos de mesa. A Tabela 3 mostra as estatísticas descritivas dos parâmetros sensoriais avaliados para cada uma das amostras de vinho tinto.

Tabela 3: Pontuação conferida aos atributos sensoriais avaliados.

\begin{tabular}{|c|c|c|c|c|}
\hline Parâmetros & Vinho A & Vinho B & Vinho $F$ & Valor $\mathrm{P}$ \\
\hline Acidez (ACD) & $6,25 \pm 1,98^{1}$ & $6,62 \pm 1,68$ & $6,50 \pm 2,56$ & 0,912 \\
\hline Corpo (COP) & $6,50 \pm 2,50$ & $7,62 \pm 2,44$ & $6,12 \pm 2,69$ & 0,389 \\
\hline Doçura (DOC) & $5,00 \pm 2,93$ & $4,87 \pm 2,35$ & $4,00 \pm 3,16$ & 0,263 \\
\hline Adstringência (ADS) & $7,12 \pm 1,12$ & $7,37 \pm 2,06$ & $7,87 \pm 2,23$ & 0,637 \\
\hline Álcool (ALC) & $7,87 \pm 1,55$ & $7,75 \pm 2,12$ & $7,37 \pm 1,76$ & 0,794 \\
\hline Cor (COR) & $8,00 \pm 1,69$ & $8,75 \pm 1,38$ & $8,37 \pm 1,68$ & 0,254 \\
\hline Odor (ODR) & $8,00 \pm 1,92$ & $7,62 \pm 3,02$ & $7,00 \pm 2,98$ & 0,408 \\
\hline
\end{tabular}

A partir dos dados da Tabela 3 foi possível observar a ausência de diferenças estatisticamente significativas quando as amostras de vinhos foram comparadas em cada um dos parâmetros sensoriais. Como não houve nenhum parâmetro que resultou em um valor $P$ inferior ao nível de significância adotado, o teste de comparação múltipla de Tukey não foi aplicado.

A análise multivariada foi aplicada com o objetivo de verificar relações entre as propriedades físico-químicas e o perfil sensorial das amostras de acordo com os atributos avaliados. Clustering Analysis mostrou a formação de dois nítidos clusters no gráfico de dispersão multidimensional, sendo o cluster 1 formado pelas propriedades físico-químicas teor de fenólicos totais, acidez volátil e extrato seco total juntamente com os parâmetros sensoriais de odor, cor, corpo e doçura; e o cluster 2 formado pelas propriedades físico-químicas acidez total e fixa, teor alcoólico e açúcar redutor, juntamente com os atributos sensoriais de acidez, álcool e adstringência (Figura 1).

A presença de determinadas propriedades físico-químicas no mesmo cluster de determinados atributos sensoriais mostra intensa relação entre esses fatores, evidenciando a influência química na resposta sensorial dos provadores. 


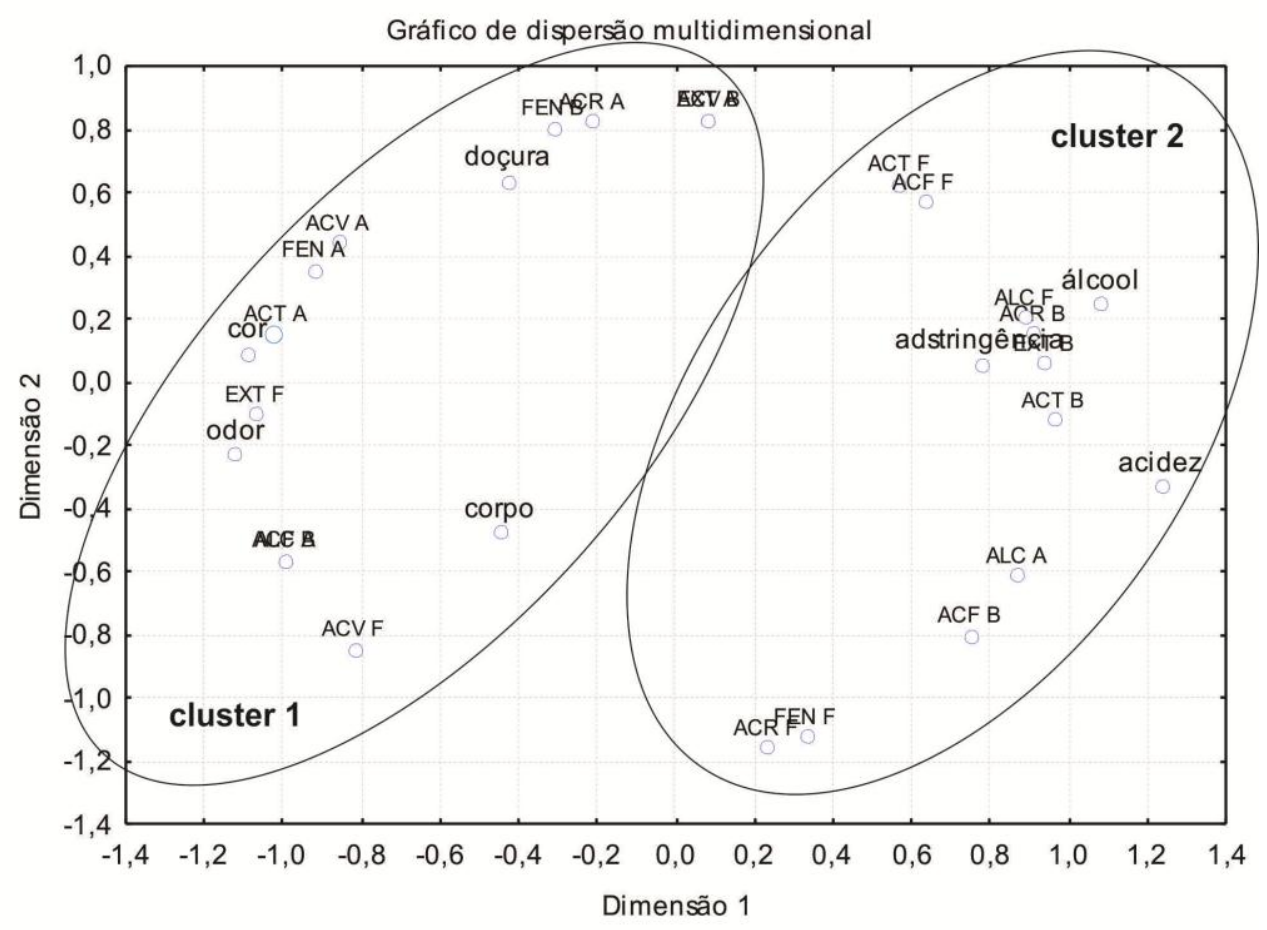

Figura 1: Gráfico de dispersão multidimensional relativo aos perfis físico-químico e sensorial.

Os resultados da Figura 1 mostraram a intensa influência da propriedade físico-química acidez volátil no odor dos vinhos, visto que a acidez volátil é caracterizada por ácidos como o acético, fórmico, butírico, propiônico e ácidos graxos com cadeias superiores a 12 carbonos. Esses ácidos graxos são metabolizados pelas leveduras e bactérias existentes no mosto, promovendo a liberação de aromas do vinho (JACKSON, 2008). Além disso, foi comprovada a relação entre o extrato seco total e o corpo dos vinhos como verificado em estudos de Yanniotis et al. (2007), independentemente do fato de o vinho ser de mesa ou fino. Adicionalmente, foi possível constatar a influência do teor de açúcar redutor do vinho de mesa $A$ na avaliação do corpo (JACKSON, 2008), visto que essa amostra apresentou o maior valor para essa determinação físico-química dentre as amostras estudadas.

A cor foi um atributo sensorial influenciado pelos teores de fenólicos totais dos vinhos de mesa, mostrando que a coloração bordô típica desses vinhos influenciou de forma crítica a avaliação desse atributo. De acordo com Manfroi (2004), os vinhos jovens de mesa, que não passam por período de envelhecimento, apresentam coloração mais avermelhada, ao passo que os vinhos finos, geralmente submetidos ao envelhecimento, apresentam complexação de antocianinas com taninos, promovendo complexidade à cor, evidenciando uma coloração mais alaranjada. Por essa razão, mostrou-se a maior atratividade dos provadores pela coloração dos vinhos de mesa e, além disso, a relação do teor de fenólicos totais do vinho fino com o atributo adstringência, como visualizado no cluster 2. Os compostos fenólicos por meio da polimerização das antocianinas com os taninos promovem a sensação bucal de adstringência (JACKSON, 2008), reiterando o resultado obtido nesse estudo.

A doçura foi influenciada pelo teor de açúcares redutores dos vinhos de mesa que, por sua vez, são caracterizados pelos açúcares não-fermentescíveis, responsáveis por determinar a sensação de doçura desses vinhos (JACKSON, 2008). A sensação de acidez e álcool avaliada pelos provadores selecionados foi relacionada com as propriedades acidez total e fixa e teor alcoólico, 
respectivamente. É possível observar que os atributos doçura, cor, odor e corpo formaram o cluster 1, ao passo que os atributos adstringência, álcool e acidez formaram o cluster 2, evidenciando o contraste entre esses clusters, visto que tal análise promove o agrupamento dos atributos avaliados pela sua similaridade.

\section{CONCLUSÕES}

Este estudo trouxe resultados sobre o perfil físico-químico e sensorial de três vinhos da região noroeste de São Paulo. Foi possível constatar a relação entre o extrato seco total e o corpo das amostras, além da influência da acidez volátil na avaliação do odor dos vinhos estudados. Além disso, foi interessante observar o comportamento do teor de fenólicos totais em relação aos atributos sensoriais, visto que se relacionou de forma direta com a cor para os vinhos de mesa e com adstringência para o vinho fino.

Todos os vinhos apresentaram perfil físico-químico de acordo com os parâmetros preconizados pela legislação brasileira, sendo importante para a avaliação da qualidade do vinho. É importante salientar a relevância do estudo das relações entre os parâmetros físico-químicos e os atributos sensoriais, visto que não é uma análise explorada nos estudos científicos e possibilita explicar alterações sensoriais por possíveis alterações nos parâmetros físico-químicos.

\section{REFERÊNCIAS}

1. AMERINE, M. A.; OUGH, C. S. Wine and must analysis. 2. ed. New York: John Wiley \& Sons, 1986. 377 p.

2. AMERINE, M.A.; BERG, H.W.; CRUESS, W.V. The technology of wine making. 2. ed. Westport: AVI, 1967. 797p.

3. AOAC. Association of Official Agricultural Chemists. Official methods of analysis of the AOAC International. Washington, 1992, 1141p.

4. BRASIL. Lei n. 10970 de 16 de novembro de 2004. Altera dispositivos da Lei n. 7678 de 8 de novembro de 1988, que dispõe sobre a produção, circulação e comercialização do vinho e derivados de uva e do vinho, e dá outras providências. DOU: Diário Oficial da União, Brasília, DF, 2004.

5. BRASIL. Portaria n. 76 de 27 de novembro de 1986. Aprova os métodos analíticos que passam a constituir padrões oficiais para análise de bebidas e vinagres estabelecidos pelo Decreto n. 73267 de 06 de dezembro de 1973. DOU: Diário Oficial da União, Brasília, DF, 1986, seção 1, p. 18152-18173

6. CAMARGO, U.A. Técnicas de produção vitícola com ciclos sucessivos em condições tropicais. In: WORKSHOP INTERNACIONAL DE PESQUISA, 1., 2004, Petrolina e Recife. Anais... Petrolina e Recife: 2004, 11p.

7. CAMPO, E.; BALLESTER, J.; LANGLOIS, J.; DACREMONT, C.; VALENTIN, D. Comparison of conventional descriptive analysis and a citation frequency-based descriptive method for odor 
profiling: An application to Burgundy Pinot Noir wines. Food Quality and Preference, v.21, p.44-55, 2010.

8. CASTILHOS, M. B. M.; CONTI-SILVA, A. C.; DEL BIANCHI, V. L. Effect of grape pre-drying and static pomace contact on physicochemical properties and sensory acceptance of Brazilian (Bordô and Isabel) red wines. European Food Research and Technology, v.235, n.2, p.345354, 2012.

9. CHAVARRIA, G.; SANTOS, H.P.; ZANUS, M.C.; ZORZAN, C.; MARODIN, G.A.B. Caracterização físico-química do mosto e do vinho Moscato Giallo em videiras cultivadas sob cobertura de plástico. Pesquisa Agropecuária Brasileira, v.43, n.7, p.911-916, 2008.

10. CHIRA, K.; PACELLA, N.; JOURDES, M.; TEISSEDRE, P. Chemical and sensory evaluation of Bordeaux wines (Cabernet Sauvignon and Merlot) and correlation with wine age. Food Chemistry, v.126, p.1971-1977, 2011.

11. DUTCOSKY, S. D. Análise Sensorial de Alimentos. 3 ed. Curitiba: Editora Champagnat, 2011.

12. FOLIN, O.; CIOCALTEAU, V. On tyrosine on triptophane determinations in proteins. Journal of Biololgy and Chemistry, v. 73, n. 2, p. 627-651. 1927.

13. GIRARD, B.; YUKSEL, D.; CLIFF, M. A.; DELAQUIS, P.; REYNOLDS, A.G. Vinification effects on the sensory, colour and GC profiles of Pinot noir wines from British Columbia. Food Research International, v. 34, p. 483-499, 2001.

14. GUERRA, C.C.; MANDELLI, F.; TONIETTO, J.; ZANUS, M.C.; CAMARGO, U.A. Conhecendo o essencial sobre uvas e vinhos. EMBRAPA Uva e Vinho - CNPUV (Documentos). Bento Gonçalves, 69 p. 2009.

15. HASHIZUME, T. Fundamentos de tecnologia do vinho. In: AQUARONE, E.; LIMA, U. A.; BORZANI, W. Alimentos e bebidas produzidos por fermentação. São Paulo: Edgard Blücher, 1983.

16. INSTITUTO ADOLFO LUTZ. Normas analíticas do Instituto Adolfo Lutz. v. 1: Métodos químicos e físicos para análise de alimentos. 4. ed. São Paulo: IMESP, 2005. 1020p.

17. JACKSON, R. S. Wine science: principles and applications. 3 ed. San Diego: Academic Press, 2008. 751p.

18. LEE, S.J.; LEE, J.E.; KIM, H.W.; KIM, S.S.; KOH, K.H. Development of Korean red wines using Vitis labrusca varieties: instrumental and sensory characterization. Food Chemistry, v.94, p.385-393, 2006.

19. MANFROI, V. Degustação de Vinhos. Porto Alegre: Editora da UFRGS, 2004, 127p. 
20. MELLO, L.M.R. Tendências de consumo e perspectivas de mercado de vinhos no Brasil. EMBRAPA Uva e Vinho - CNPUV, Bento Gonçalves, 7p. 2002.

21. ORTEGA-HERAS, M.; PÉREZ-MAGARIÑO, S.; CANO-MOZO, E.; GONZÁLEZ-SAN JOSÉ, M.L. Differences in the phenolic composition and sensory profile between red wines aged in oak barrels and wines aged with oak chips. Food Science and Technology, v.43, p.1533-1541, 2010.

22. RIZZON, L.A. Sistema de produção de vinho tinto: Recebimento da uva. Sistemas de Produção - EMBRAPA Uva e Vinho - CNPUV. Bento Gonçalves, 2006.

23. THORNGATE, J. H. The physiology of human sensory response to wine: A review. American Journal of Enology and Viticulture, v. 48, n. 3, p. 271-279, 1997.

24. TONIETTO, J.; CAMARGO, U.A. Vinhos tropicais no Brasil e no mundo. Bon Vivant, v.8, n.94, p.15, 2006.

25. YANNIOTIS, S.; KOTSERIDIS, G.; ORFANIDOU, A.; PETRAKI, A. Effect of ethanol, dry extract and glycerol on the viscosity of wine. Journal of Food Engineering, v.81, p.399-403, 2007.

26. ZOECKLEIN, B.W.; Fugelsang K.C., Gump, B.H.; Nury, F.S. Wine analysis and production. New York: Chapman \& Hall, 1994, 621 p. 